\title{
Introduction to special issue on sustainable computing for ultrascale computing
}

It is our pleasure to serve as guest editors of this special issue on Sustainable Computing for Ultrascale Computing of Sustainable Computing: Informatics and Systems (SUSCOM).

The growth of infrastructures towards exascale (and beyond) is on the way. Today only the most sustainable and energy-efficient solutions will make it a reality, in order to keep an upper limit of energy consumption at a reasonable level (somewhere around 20 MW of electrical power for an exascale supercomputer, as requested by the Department of Energy in USA). Besides environmental concerns, the increasing costs for electricity advocate for finding innovative and disruptive solutions to power these infrastructures.

In this special issue, we select and present original works focused on addressing new research and development challenges, developing new techniques and advanced information technology solutions in sustainable computing in the age of ultrascale (exascale and beyond), from infrastructure and machine architectures, middleware, applications, runtime and programming point of view.

Aligning with the high-quality standards of SUSCOM, we were highly selective and accepted only the top contributions in this special issue after a rigorous review process, with several round of reviewing.'The selected papers reflect key issues related to green computing and energy efficiency at different levels, including energy monitoring, power estimation through modelling, energy and performance metrics, parallel processing and scheduling, and the usage of renewable energies.

The accepted papers are introduced below:

In future large scale machines, monitoring the power consumption will raise challenges for collecting, processing and storing values when thousands of heterogeneous resources will be shared. The paper " Energy Monitoring as an Essential Building Block Towards Sustainable Ultrascale Systems " surveys energy monitoring of large-scale systems and methodologies for monitoring the power consumed by large systems. It discusses challenges to be addressed towards monitoring and improving the energy efficiency of ultra scale systems. The authors discuss potential gaps in existing solutions when tackling emerging large-scale monitoring scenarios.

Besides monitoring the energy consumption, one possibility is to use models to estimate it. In the paper " Understanding Hardware and Software Metrics with respect to Power Consumption ", the authors utilize statistical techniques to investigate the usage of hardware counters as potential indicators of energy behavior. Their idea is to capture hardware counters, software counters, and power, and to identify interesting behavior while reducing the amount of data to capture. The identified candidate counters are used for building linear models for power prediction with a relative accuracy of $3 \%$.

The paper « Performance and Energy Metrics for Multi-threaded Applications on DVFS Processors " combines thread-level parallelism and frequency scaling (in fact a scaling factor on speed). The authors propose several metrics: For instance, the energy speedup metric related to the difference in energy consumption using one or several threads, at a fixed frequency; the energy reduction factor when using different frequencies; an energy per speedup metric; a power speedup; a power increase factor; ... Extensive analysis on PARSEC and SPLASH-2 benchmarks are given and the usage of the novel metrics advocated.

A common method to create parallel program is to divide tasks in subtasks, recursively until the it is not any more efficient to divide, on a given architecture (i.e. given the number of cores). The paper " Understanding the impact of task granularity in the energy consumption of parallel programs " analyses several cut-off methods to stop the spawning of new tasks at the right moment, and proposes selected novel algorithms. Experiments on real platforms and programs show the influence of several parameters on both execution time and energy. From these, the authors conclude on the best choices for performance, energy savings and good tradeoffs.

In the paper " Power-efficient Scheduling of Parallel Real-time Tasks on Performance Asymmetric Multicore Processors ", the authors address real-time power-aware scheduling problem of parallel tasks on a performance asymmetric multicore processor. They propose to divide tasks on the available cores and execute them with a classical EDF (Earliest Deadline First) algorithm, together with DVFS (Dynamic Voltage Frequency Scaling). Their resulting algorithm (ParallelEDF) show better performance when utilisation is high, and is formally modelled and verified using High Level Petri Nets, Satisfiability Modulo Theory and Z3 Solver.

In the article « Feasibility of Using Renewable Energy to Supply Data Centers in 60 Degrees North Latitude ", the authors study the possibility of using renewable energy according to the geographical location of the datacenter. The key study is for the city of Turku, Finland. They introduce a metric called Minimum Percentage Supply, which represents the fraction of the total data center energy consumption that renewable energy, produced by one wind tur- 
bine and one solar panel, can cover. They conduct a feasibility for a data center powered by renewable energy on such a location. While such an approach is not yet feasible for exascale computers, we see several projects worldwide using renewable energy that can, at least partly, reduce the $\mathrm{CO} 2$ emissions in the usage of ICT.

We hope that the readers will like the papers in this special issue as much as we liked selected them, and that the scientific insights are informative and useful. The guest editors wish to thank the authors of all submitted manuscripts, without whom this special issue would not have been possible. They also thank the reviewers who provided a thorough evaluation of the submitted manuscripts in a timely manner. We appreciate the assistance of the Special Issues Editor-in- Chief, Behrooz Shirazi, and the SUSCOM editorial staff throughout the process of bringing out the special issue.
The guest editors finally would like to thank the European IC1305 COST Action called NESUS, "Network for Sustainable Ultrascale Computing" (www.nesus.eu), whose inspiration gave birth to this special issue.

Laurent Lefèvre Inria, Ecole Normale Supérieure of Lyon, University of Lyon, France

Jean-Marc Pierson

IRIT, University of Toulouse, France E-mail addresses: laurent.lefevre@inria.fr(L. Lefèvre), pierson@irit.fr (J.-M. Pierson). 